\title{
THE HEART RATE WITH EXERCISE IN PATIENTS WITH AURICULAR FIBRILLATION
}

\author{
BY \\ J. A. C. KNOX \\ From the Department of Physiology, King's College, London
}

Received December 14, 1948

In a previous article (Knox, 1940) an accurate method of recording the changes in heart rate during exercise was described, and results were given for normal subjects performing a brief light step-test. This technique has now been applied to patients with auricular fibrillation. The present series consists of twenty-two tracings from thirteen ambulant patients with fibrillation.

The standard exercise was five ascents of two steps each ten inches high, the rate of stepping being 96 a minute. The exercise began and ended in the sitting posture and its total duration was about twenty seconds. The heart beats were electrically recorded on a smoked drum throughout exercise and were counted in five-second periods to the nearest tenth of a beat.

The clinical condition of the patients with fibrillation was classified as good, fairly good, fair, or poor on the basis of the functional capacity classification of the American Heart Association (1926). This was called the patients' "Exercise Tolerance Group." In addition, the patients were also grouped according to whether they were receiving digitalis or not.

Full details of the method of calculating the various heart rate indices were given in the previous article and only a summary need be given here.

The initial rate is the heart rate averaged over the ten seconds immediately preceding exercise.

The maximum rate is the highest rate observed during exercise averaged over a five-second period.

The acceleration of the heart rate is calculated by subtracting the rate in the five seconds immediately preceding exercise from the maximum rate, and dividing the result by the time taken to reach the maximum rate.

The post-exercise rate is the heart rate averaged over a thirty-second period beginning five seconds after exercise ends.

\section{RESULTS}

The heart-rate indices. The figures for the various heart-rate indices of the fibrillation cases in the different "Exercise Tolerance Groups" are shown in Table I. For comparison, the mean results for 100 normal subjects $(75$ men and 25 women) are also included in this table. It can be seen that there was no correlation between any of these heart rate indices and the patient's clinical condition. This was not unexpected, as the number of patients in each group is very small and in addition the completely irregular ventricular rhythm present in fibrillation produces sudden arbitrary changes in heart rate in the successive five-second intervals counted. Certain general characteristics, however, can be seen in Table I.

Apart from the single case with good tolerance the maximum rates reached during the step-test were much higher than in the normal subjects. This was also true for the percentage and actual increases over the initial rates, and for the postexercise rates.

The curve of heart rate during exercise. Fig. 1 shows the mean curves of heart rate during exercise for the different tolerance groups in all the fibrillation patients who were on digitalis. For comparison, the mean curve of the normal subjects is also included in the figure. Two of the curves (fairly good and fair) show a distinct fall in heart rate during the first five seconds of the exercise, a phenomenon that is of course never seen in normal subjects. In general, compared with the normal curve, the acceleration immediately after the beginning of exercise in the digitalized patients with fibrillation is less; but later during the exercise great acceleration occurs, reaching a higher value than in the normals. Thus in Fig. 1 all the fibrillation curves show a steeper slope in some portion of their course 
TABLE I

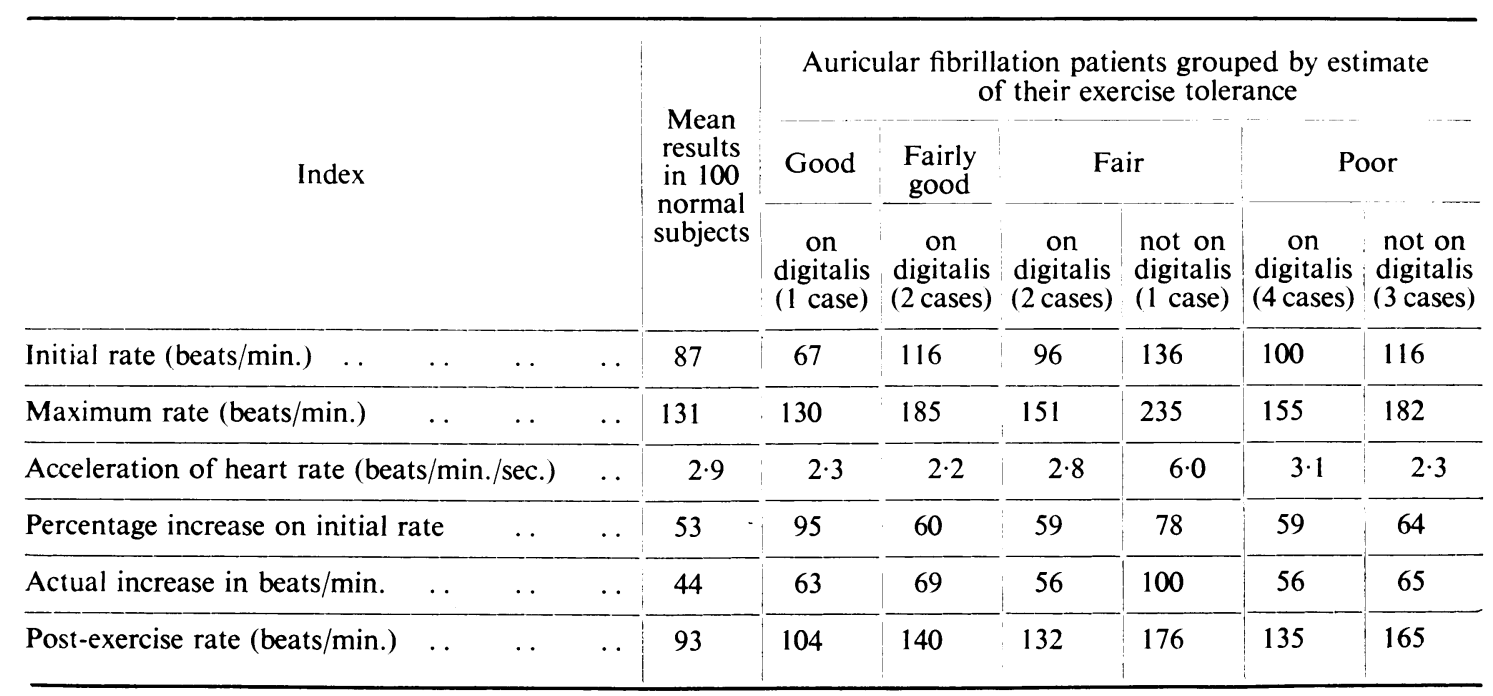

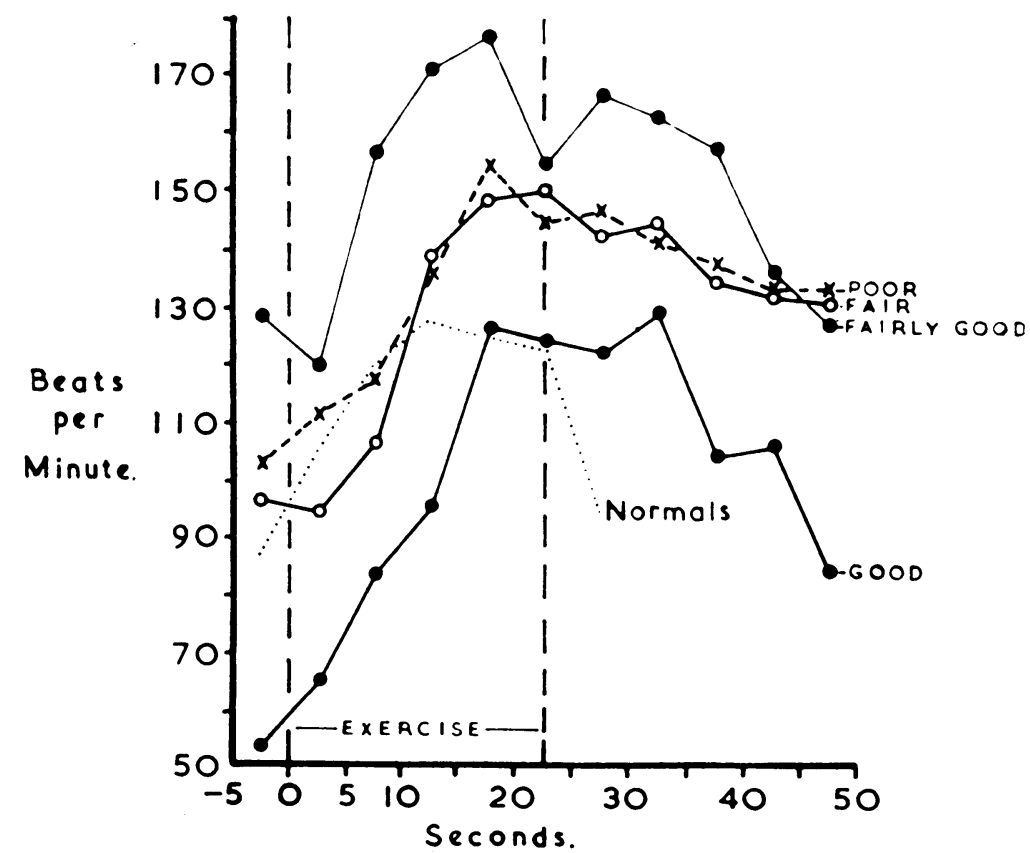

FIG. 1.-Auricular fibrillation patients on digitalis (Series C). Graphs of mean heart rates before, during and after the standard step test in patients classified into their different " tolerance groups."

The dotted line shows the mean curve for normal subjects. 


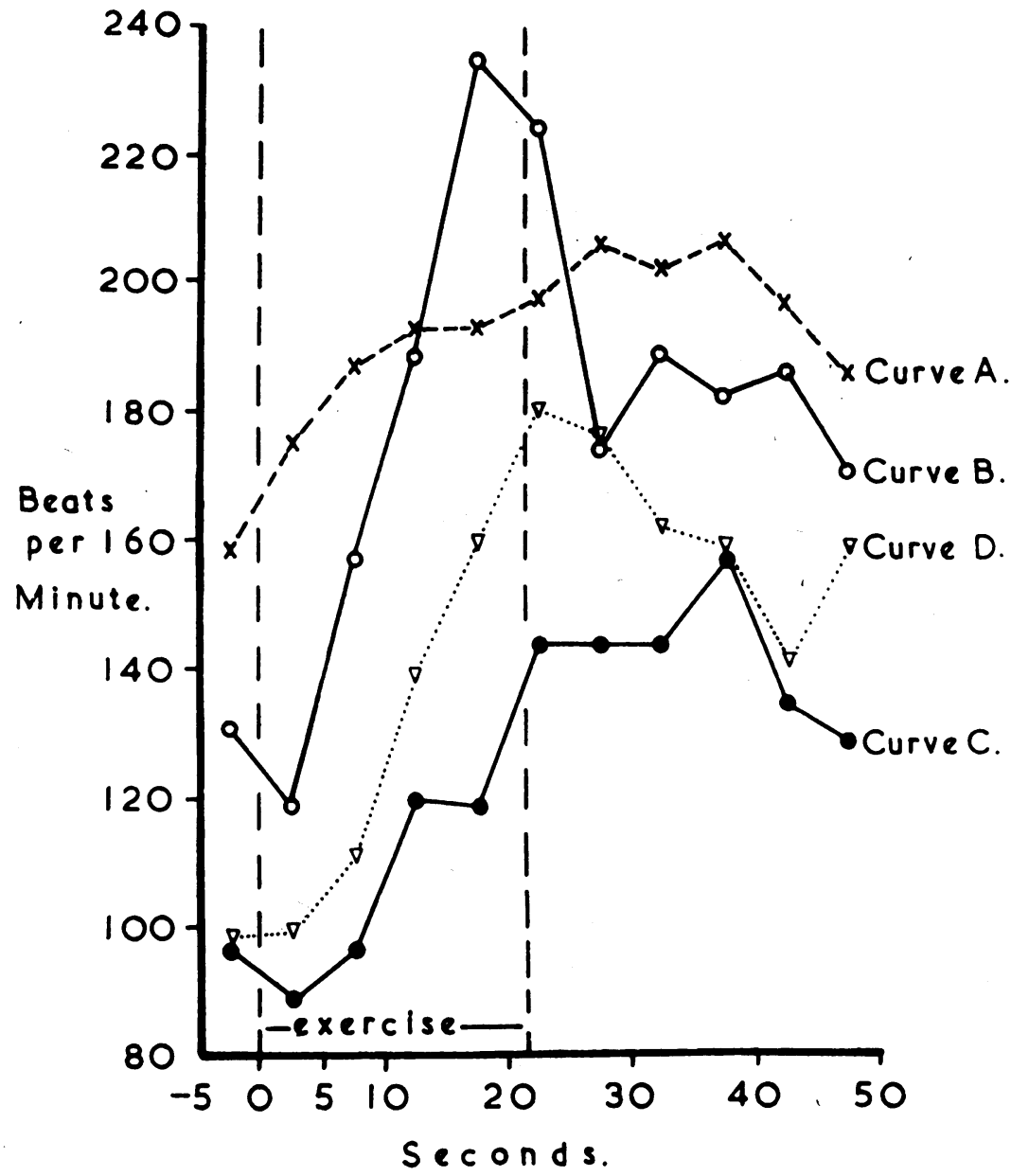

FIG. 2.-Auricular fibrillation patients not on digitalis (Series C). Graphs of heart rate before, during and after the standard step test in four patients.

Curves A, B, C, and D are each from a separate patient.

than does the normal curve. This sudden delayed acceleration was very characteristic of those with fibrillation. It will also be seen that after the end of exercise the heart rate falls very slowly compared with the normal, especially in the poorer tolerance groups.

The curves of heart rate during the step test in four patients with fibrillation who were not on digitalis are given in Fig. 2. In general they show the same characteristics as the digitalized patients; these include the drop in rate at the beginning of exercise (in two cases), the delayed acceleration (in three cases) and the continuation of a high heart rate after the end of exercise. Curve $B$, from a patient with mitral stenosis and fair tolerance has the highest maximum rate reached by any patient, 235 beats a minute averaged over a five-second period. A portion of the actual tracing is given in Fig. 3. It is surprising that such an extreme frequency was reached during a very mild exercise lasting only twenty seconds in a patient who could get about reasonably well. In both the digitalized and non-digitalized groups the curves of heart rate for the patients with " poor" tolerance did not as a rule show such high instantaneous values of acceleration as the other tolerance groups.

Variations in a single subject. It might be expected that owing to the complete irregularity of the ventricular rate in fibrillation there would be very great variations in the shape of the heart rate curve, even in the same patient on the same day. Fig. 4 shows three curves of the response of the heart rate to the standard step test performed at 15 minute intervals by the same patient with fibrillation. The 
SIGNAL

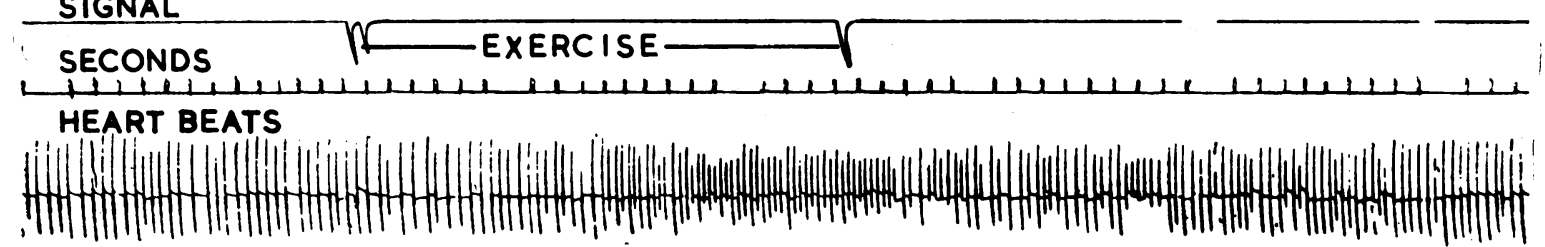

\section{- RESPIRATION}

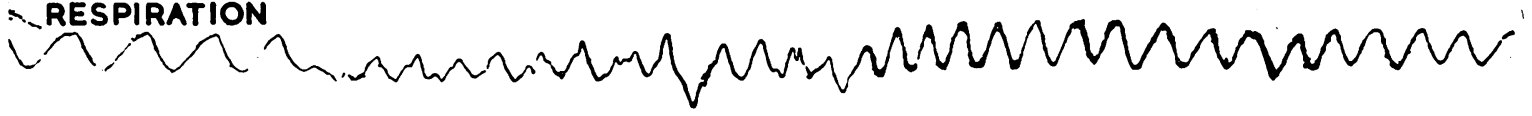

FIG. 3.-Kymograph tracing of heart beats from a patient with auricular fibrillation. Between the dips in the signal line the patient performed the standard two-step test. During the last half of exercise the extremely high heart rate of 235 beats a minute was attained.

Upper line -signal

Second line - time in seconds
Third line - heart beats

Fourth line -respiration

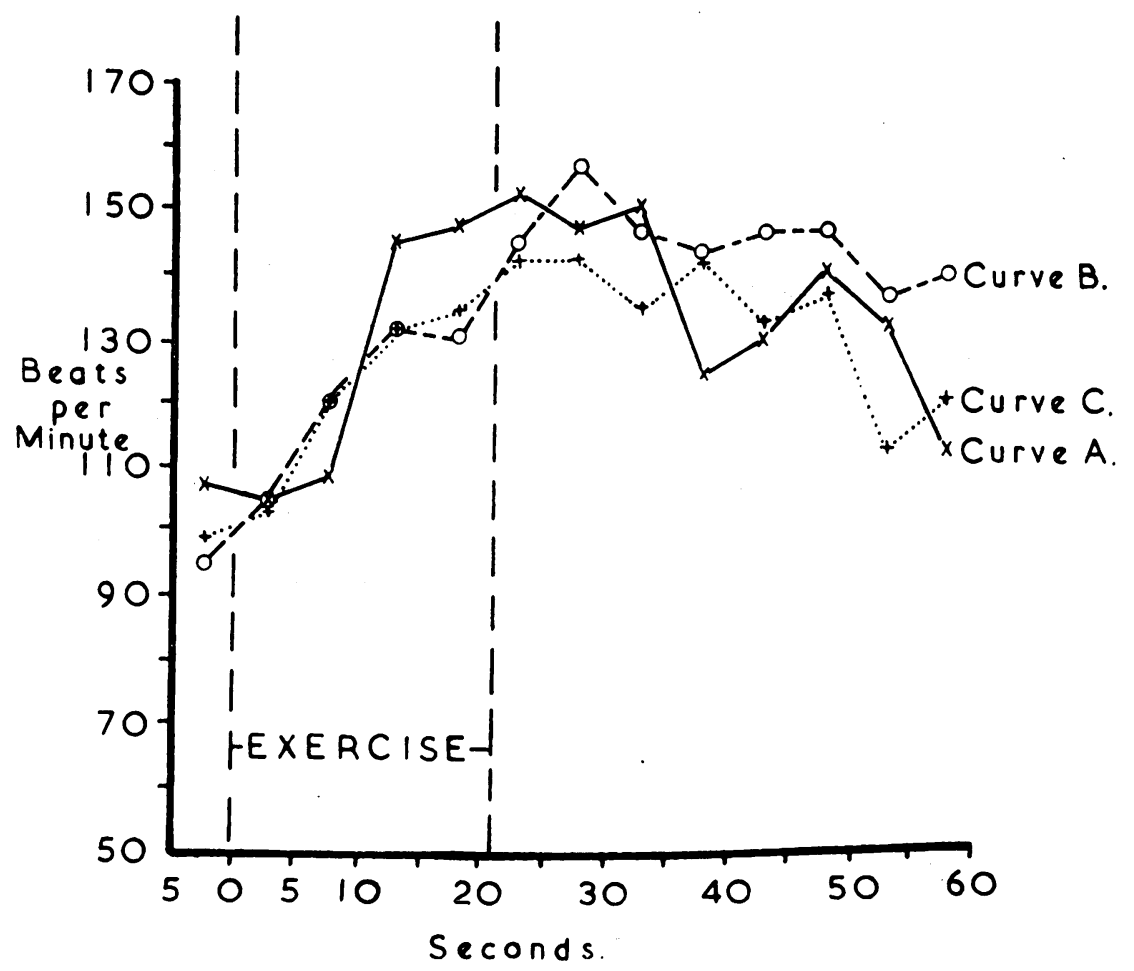

FiG. 4. -Effect of repetition of the standard step-test 3 times at 15-minute intervals on the heart rate of a patient with auricular fibrillation (Series C, Case C 49).

Curve A-Heart rate before, during, and after the standard step-test.

Curve B-The same, 15 minutes later.

Curve C-The same, 15 minutes after Curve B. 
curves were taken in the order A, B, C. The variation is greater than one would expect from a normal subject, but in view of the complete arrhythmia the general shape of the curve is remarkably well maintained. It can be seen that the acceleration tends to decrease in successive tests. Similar results were obtained from two other patients with fibrillation.

There was some evidence that this constancy in the shape of the heart rate curve of a given patient may persist over considerable periods of time even when the clinical condition has improved or deteriorated. The curves for two such patients during the standard step test are shown in Fig. 5. Curve A is from a patient on digitalis whose tolerance classification was "fairly good." Curve B is from the same patient one year later when the clinical condition had improved, the dosage of digitalis remaining unchanged. Curve $\mathrm{C}$ shows the response in another patient with fibrillation, also on digitalis. His tolerance classification was "poor" and coupling of the beats was present. Curve D is from the same patient six months later when his general condition had become worse, though the coupling was now absent. The figure demonstrates how the characteristic shape of the curve for each patient was maintained even after an interval of many months. Thus curves A and B both show a "peak" type of curve with marked oscillations after the end of exercise, whereas curves $C$ and $D$ are of the "plateau" type with a relatively smooth postexercise fall in rate.

The improvement in the condition of the first patient is accompanied by a fall in the general level of his curve, whereas the reverse is true of the second patient whose condition had become worse. While this does suggest that the exercise heart rate curves of these patients may vary with their clinical condition, it is also possible that the variations were due to different degrees of digitalization, although the actual dosage remained constant in each case.

The tracing from which curve $C$ was taken showed the actual onset of a period of coupling. A reproduction of part of this tracing is given in Fig. 6, and the start of coupling in a burst of extrasystoles can

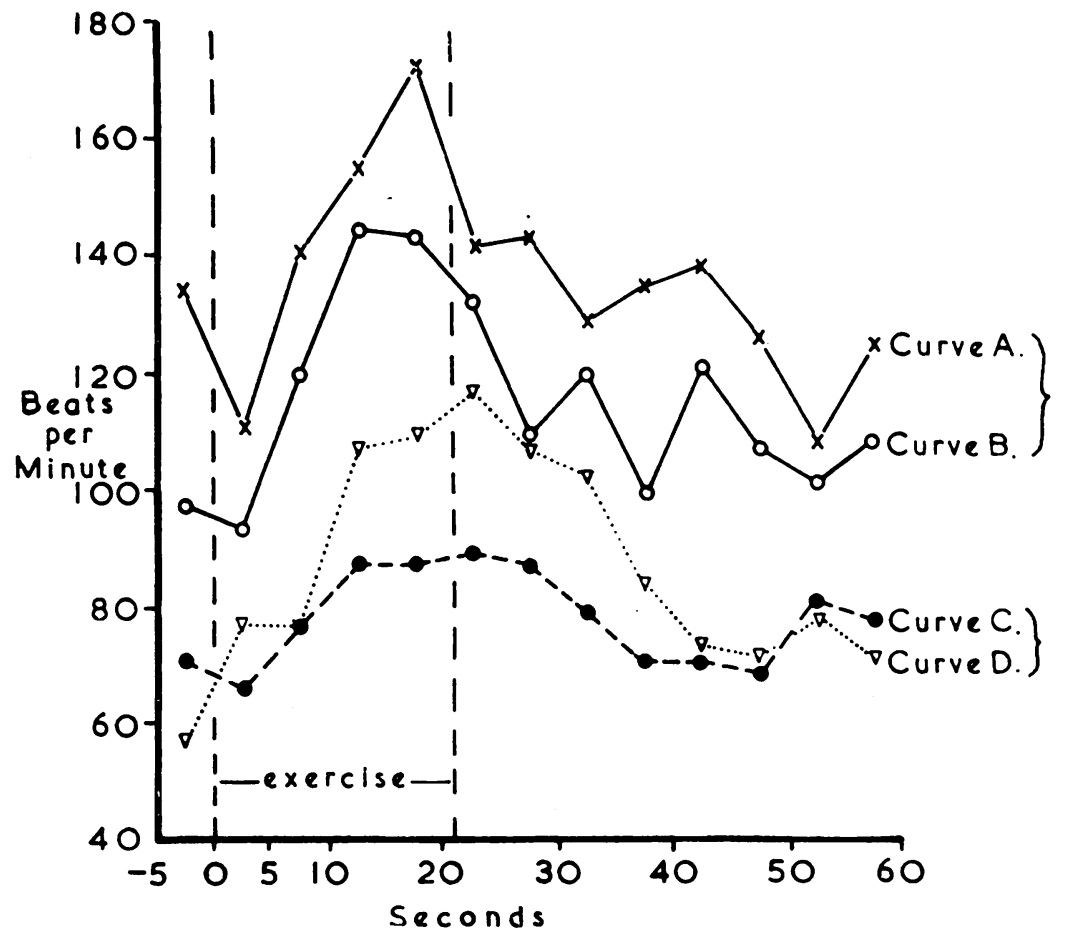

Fig. 5.-Curves of heart rate during the standard step-test in two cases of auricular fibrillation.

Curve A-Heart rate before, during, and after the step-test.

Curve B-The heart rate in the same patient one year later, when the clinical condition had improved.

Curve C-Heart rate before, during, and after the step-test in another fibrillation patient.

Curve D-The heart rate six months later in the same patient as Curve $\mathrm{C}$, when the clinical condition had deteriorated. 


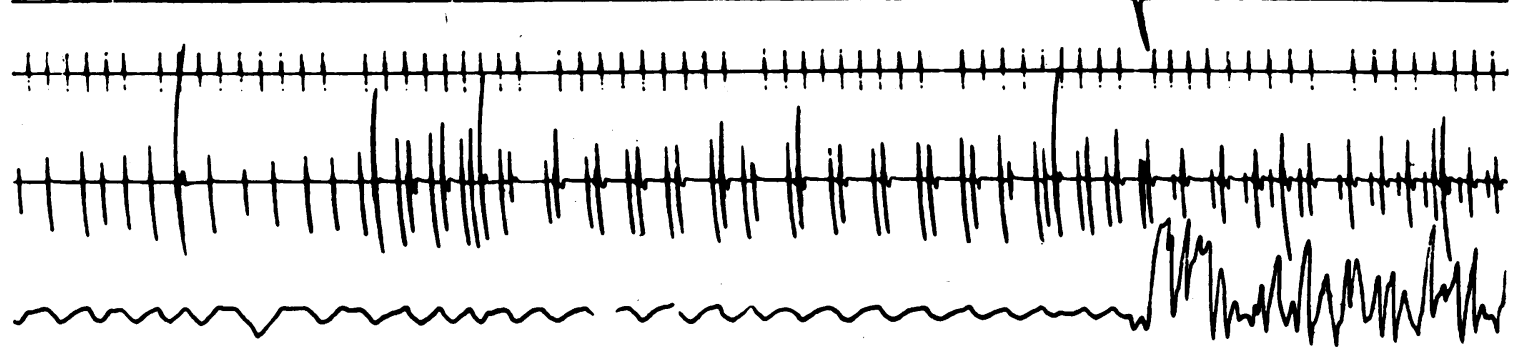

FIG. 6.-Kymograph tracing showing onset of a period of coupling in a patient with auricular fibrillation.

The coupled beats begin after a burst of extrasystoles.

The dip in the signal line indicates the beginning of the standard step-test.

$$
\begin{aligned}
& \text { Upper line - signal } \\
& \text { Second line-time in seconds }
\end{aligned}
$$

be seen to occur about thirty-five seconds before the beginning of exercise. The coupled beats persisted throughout exercise and were still present when the patient left the laboratory some fifteen minutes later. This patient may have been of the type referred to by Goodman and Gilman (1941) in whom relatively small doses of digitalis may cause coupling, as he was receiving only 1 grain tab. dig. pulv. once daily.

\section{Discussion}

Blumgart (1924) was the first to attempt a quantitative estimate of the effect of exercise on the heart rate in cases of fibrillation. His exercise consisted in stepping twenty times on and off a chair, and the test was carried out by six controls and nine patients. Heart rates were recorded before and immediately after exercise, but no records were obtained during the exercise period. He concluded that fibrillating hearts respond to a given exercise by a disproportionate rise in ventricular rate and by a delayed return to normal. Both these findings are upheld by the present results as can be seen from Fig. 1 and 2.

Blumgart also found that digitalis in ordinary doses failed to prevent the exaggerated response to exercise; in fact, the actual increase in rate was slightly greater under digitalis. In the present series it was not possible to compare the results in the same patients while on and off digitalis but it can be seen from Table I that the actual increase in heart rate of the digitalized cases was much greater than in the normals so that here also digitalis has failed to protect completely against an exaggerated rise in rate. The actual increase, however, in the non-digitalized patients is on the average consider-
Third line - heart beats

Fourth line - respiration

ably greater than in patients of the same tolerance group who were receiving the drug. In general, if we consider the patients in the "poor tolerance" group of whom four were on digitalis while three were not, all the indices except the acceleration of the heart rate are higher in the non-digitalized group. Digitalis, therefore, while failing to abolish the exaggerated increase in rate, did appear to diminish it to a considerable extent in my cases. * Although contrary to Blumgart's findings, this is in agreement with the results of Weinstein, Plaut, and Katz (1940) who showed that digitalis when used in large therapeutic doses lessened the ventricular acceleration due to a standard exercise test in ambulant fibrillation cases.

This is of interest in connection with the work of Gold et al. (1939) who analysed the relative importance of the vagal and extra-vagal mechanisms by which digitalis slows the ventricle in fibrillation. They found the slowing caused by small doses of digitalis (up to about 60 per cent of the full dose) could be counteracted by large doses of atropine, so that it was largely due to vagal stimulation. When full doses of digitalis were given, atropine could no longer increase the ventricular rate and the slowing was then clearly due to extravagal actions of digitalis. The main extravagal action was said to be the increase in the refractory period of the A-V conduction system.

In 1941, Modell, Gold, and Rothendler applied these results to the exercise acceleration of the ventricle in patients with fibrillation. They concluded that in the average case the exaggerated acceleration during exercise was due chiefly, if not entirely, to decrease in vagat tone, and that blocking the vagus by atropine accelerated the ventricles to the same maximum level as extreme physical

* Dr. Maurice Campbell informs me that he (and no doubt other cardiologists) have been in the habit of teaching for years that the amount of digitalis needed for satisfactory control for a patient at rest in hospital is smaller than the amount often needed to control his heart rate when he is doing more and getting about outside the hospital. This fits in well with the experimental points. 
exertion. Extra-vagal digitalization with large doses prevented the exaggerated response to exercise through direct action on the A-V conducting system, in accordance with the theory. They also pointed out that the ventricular rate at rest does not indicate whether digitalis has caused slowing by the vagal or the extra-vagal mechanism, but that there are two simple ways of detecting extravagal digitalization. Either $2 \mathrm{mg}$. of atropine may be given intravenously or the patient may be made to exercise. If neither of these procedures raises the ventricular rate to over 100 a minute, then enough digitalis has been given to cause slowing by the extra-vagal mechanism. Judged by the latter test, the extra-vagal mechanism can only have been prominent in one of the digitalized cases of the present series and there it was associated with marked coupling of beats. It is noteworthy that this was the only case in which no delayed acceleration of the heart occurred and it is thus probable that a sudden diminution in vagal tone was the main factor causing the delayed acceleration.
SUMMARY

In a series of thirteen ambulant patients with fibrillation the maximum heart rates reached during a short standard step-test were much higher than in normal subjects.

No correlation was found between any of the heart-rate indices (initial rate, maximum rate, acceleration, percentage increase, actual increase, and post-exercise rate) and the clinical condition of the various patients.

In spite of the complete irregularity of the ventricular rate, the response to exercise in a given patient was reasonably constant.

In some cases a brief fall in heart rate occurred at the beginning of exercise.

An almost constant feature was a sudden delayed acceleration of the heart rate commencing about twelve seconds after exercise had begun.

Digitalis, while failing to abolish the exaggerated increase in rate, appeared to diminish it to a considerable extent.

\section{REFERENCES}

American Heart Association (1926). Amer. Heart J., 2, Knox, J. A. C. (1940). Brit. Heart J., 2, 289. 202. $\mathrm{H}$ (1924). Heart, 11, 49.

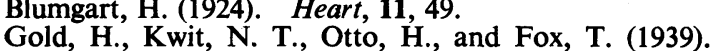
J. Pharmacol., 67, 224.

Modell, W., Gold, H., and Rothendler, H. H. (1941). J. Amer. med. Ass., 116, 2241.

Weinstein, W., Plaut, J., and Katz, L. N. (1940). Amer. J. med. Sci., 199, 498.

Goodman, L., and Gilman, A. (1941). The Pharmacological Basis of Therapeutics. New York, Macmillan, p. 520. 